Sādhanā Vol. 29, Part 2, April 2004, pp. 237-247. @ Printed in India

\title{
Performance evaluation of multi-sensor data fusion technique for test range application
}

\author{
SHRABANI BHATTACHARYA and R APPAVU RAJ \\ Integrated Test Range, DRDO, Chandipur 756 025, India \\ e-mail: \{shrabani_ju95, appavuraj\}@yahoo.com
}

\begin{abstract}
We have adopted the state-vector fusion technique for fusing multiple sensors track data to provide complete and precise trajectory information about the flight vehicle under test, for the purpose of flight safety monitoring and decisionmaking at Test Range. The present paper brings out the performance of the algorithm for different process noise and measurement noise using simulated as well as real track data.
\end{abstract}

Keywords. Multi-sensor data fusion; Test Range application; trajectory information; flight safety.

\section{Introduction}

In the context of target tracking and estimation, multi-sensor data fusion (MSDF) is the process of combining evidence/data from redundant and/or complementary sensors, to generate complete and precise information regarding location and identity of unknown numbers of unknown targets of different types. It is not possible to deduce a comprehensive picture about the entire target scenario from each of the pieces of evidence alone, due to the inherent limitations of technical features characterizing each sensor.

In a Test Range environment, continuous monitoring of the position, velocity and acceleration of the flight vehicle under test is essential for minimizing the risk to life and property resulting from an errant vehicle. This is accomplished by using the data provided by various tracking sensors. Tracking sensors deployed by us are of different types, which include RF sensors like radar and telemetry, electro-optical trackers, IR trackers and on-board sensors, i.e., inertial navigation system (INS), global positioning system (GPS) etc. Each of the sensors has its own limitations in spatial and temporal coverage. For example, acquisition and tracking for radar and electro-optical tracking system (EOTS) depend upon line-of-sight of the instrument to target. Tracking beyond the radio horizon is not feasible with RF sensors. Performance of some sensors is affected by errors like multi-path, side-lobe tracking, atmospheric effect etc. Hence, a single sensor alone cannot provide a complete assessment of actual performance of a flight vehicle from lift-off to impact.

A partial list of symbols is given at the end of the paper 
In this context, MSDF plays a crucial role in providing improved probability of detection, extended spatial and temporal coverage, reduced ambiguity and improved system reliability and robustness (Hall 1992). Among the various techniques available for MSDF, Kalman Filtering-based approach is used for the present case, as it proves to be an efficient recursive algorithm suitable for real-time application using digital computers. Among different approaches for Kalman Filter-based sensor fusion, two commonly employed techniques are (i) state-vector fusion and (ii) measurement fusion (Gan \& Harris 2001). The state-vector fusion method uses covariance of the filtered output of individual noisy sensor data to obtain an improved joint state estimate. On the other hand, the measurement fusion method directly fuses the sensor measurements to obtain a weighted or combined measurement and then uses a single Kalman Filter to obtain the final state estimate based on the fused measurement. The two philosophies are enumerated in figure 1 . However, both the systems have their own merits and demerits. The measurement fusion method, which combines multi-sensor data using minimum mean square error estimate, requires that the sensors should have identical measurement matrices. Although the measurement fusion method provides better overall estimation performance, state-vector fusion has lower computational cost and possesses the advantage of parallel implementation and fault tolerance. Judicious trade-off between computational complexity, computational time and numerical accuracy has to be made for selection of algorithm for practical application.

In the present case, state-vector fusion methodology has been employed since our tracking sensors at the Integrated Test Range, Chandipur have different tracking technologies and have diversified measurement noise characteristics. The aim is to yield three fused outputs of position and velocity of the flight vehicle based on the tracking data of twelve sensors (i.e., three radars, three telemetry (TM), four EOTS, INS and GPS).

(a)

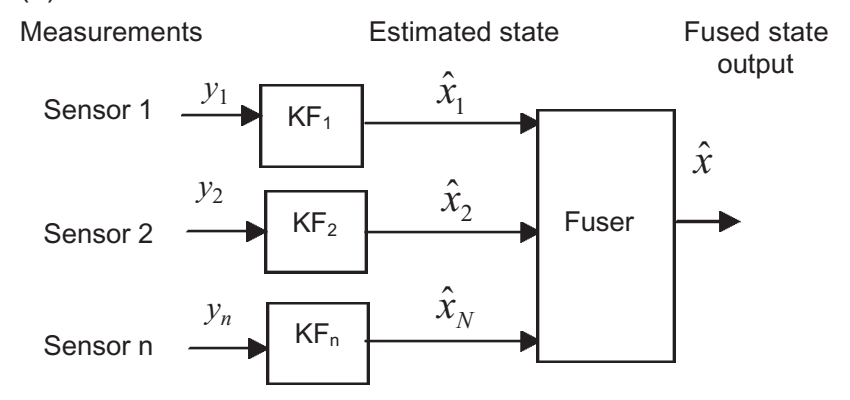

(b)

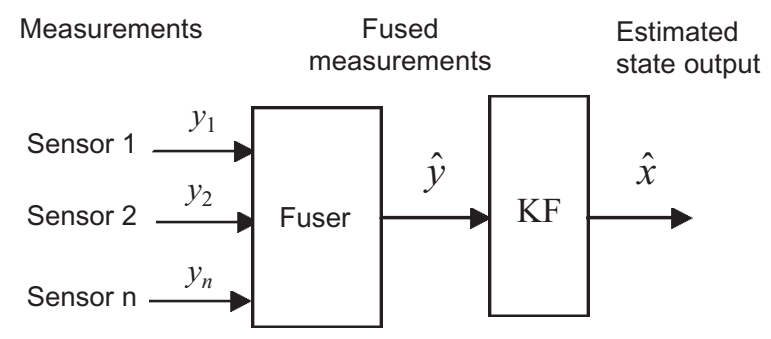

Figure 1. (a) State-vector fusion; (b) measurement fusion. 


\section{Multi-sensor data fusion strategy at Test Range}

The fusion scheme adopted at Integrated Test Range is shown in figure 2. The scheme is evolved based on the following criteria (Banerjee et al 2000):

(i) Fusion of similar type of sensors (i.e., S band-S band, EOTS-EOTS etc.);

(ii) Fusion for dissimilar type of sensor (i.e., INS-GPS, TM-S band etc.);

(iii) Priority based source selection based on

(a) Most accurate track data depending on inherent spatial and temporal coverage of the sensor, and

(b) reliability of the sensor

The fusion algorithm generates three sets of fused position and velocity estimates as output. The data from S-band radars (after conversion from Polar coordinate to Cartesian coordinate) are combined to give fused (similar sensor fusion) data set-1 as per fusion logic shown in figure 3. When data from both the radars are valid, a fused output is produced. If any one radar is valid, it is given as output. In case of no radar track, the system declares track-loss.

The INS and GPS are fused (dissimilar sensor fusion) together to yield fused data set-2 based on the philosophy shown in figure 3. In this case fused output is obtained if both the sensors are valid. Else, it provides output of single valid sensor data or declares track-loss if both sensors are invalid.

The angular data (azimuth and elevation) from telemetry stations (TM1, TM2, TM3) are combined together by means of least squares triangulation to generate position information in the Cartesian coordinate system. Similarly, angular information from the four EOTS is also triangulated. The triangulated telemetry and EOTS data, and the data from PCMC radar and other radar operating in skin mode, are fused using priority logic as mentioned in figure 4 .

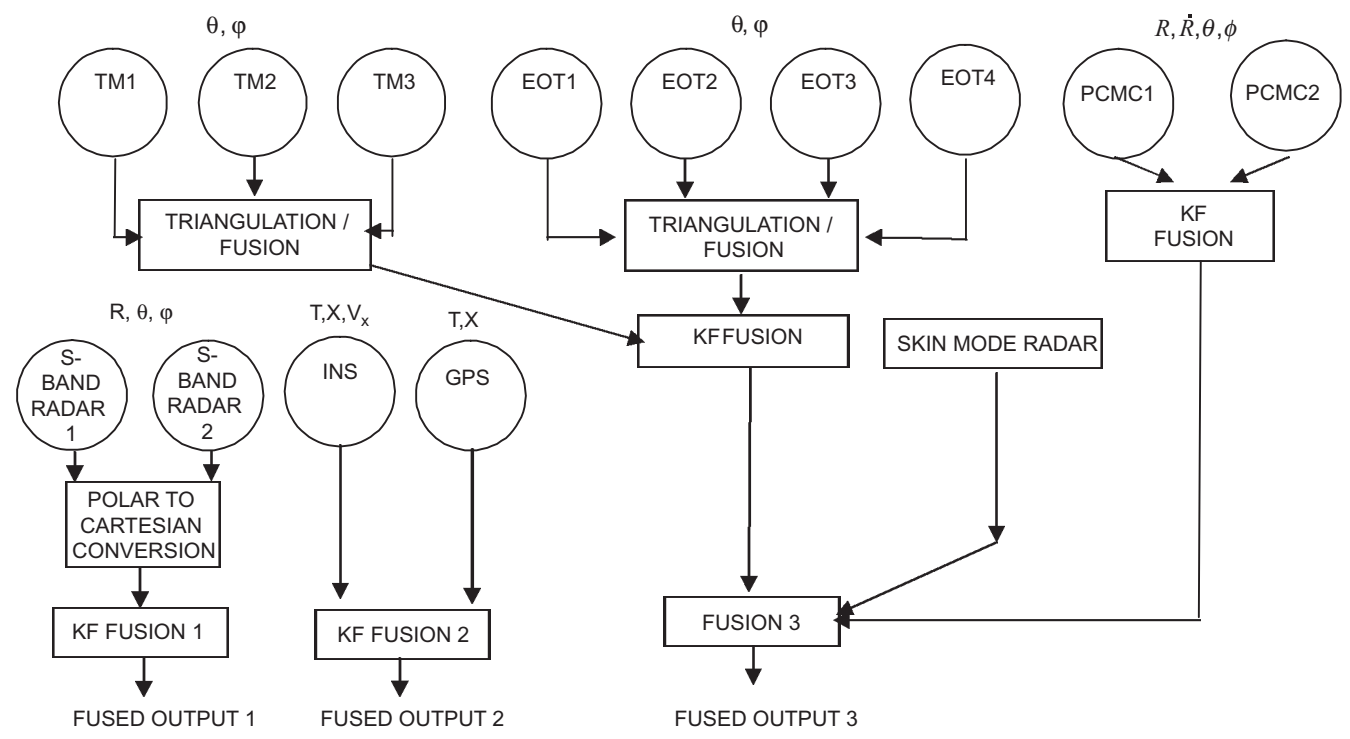

Figure 2. Fusion scheme adopted at Test Range. 
Data from Sensor 1 \&

Sensor 2

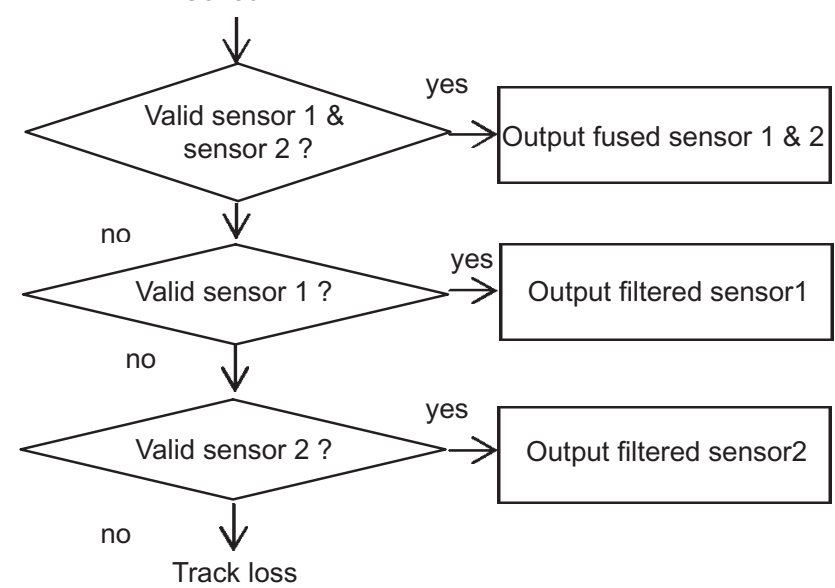

Figure 3. Algorithm of KF fusion 1 and 2 modules.

The fusion method used for the fused data sets $1,2 \& 3$ is based on state-vector fusion. In this method, individual sensor data is filtered using UD-factored Kalman filter (Girija et al 2000) using measurement noise characteristics of the respective sensors. The state and stateerror-covariance estimates of Kalman filter $(\mathrm{KF})$ for each of the sensors are then used to obtain the fused state according to the following equations (Saha 1996):

Fused state: $\hat{x}_{F i, i}=\hat{x}_{1 i, i}+\hat{P}_{1_{i, i}}\left(\hat{P}_{1_{i, i}}+\hat{P}_{2_{i, i}^{T}}^{T}\right)^{-1}\left[\hat{x}_{2_{i, i}}-\hat{x}_{1_{i, i}}\right]$,
Covariance of fused state: $\hat{P}_{F_{i, i}}=\hat{P}_{1_{i, i}}-\hat{P}_{1_{i, i}}\left(\hat{P}_{1_{i, i}}+\hat{P}_{2_{i, i}}^{T}\right)^{-1} \hat{P}_{1_{i, i}^{T}}^{T}$,

Data from EOT, PCMC, TM,

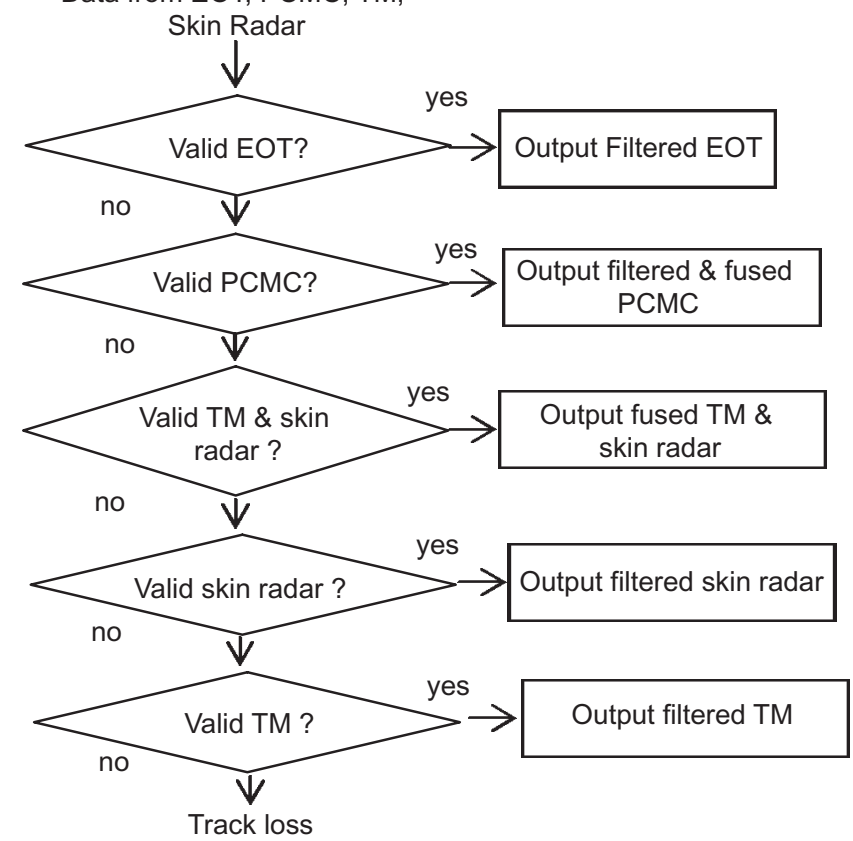

Figure 4. Algorithm of fusion 3 module. 
where

$$
\begin{aligned}
& \hat{x}_{1_{i, i}}=\text { estimated state of sensor } 1 \text { at } i \text { th instant, } \\
& \hat{x}_{2_{i, i}}=\text { estimated state of sensor } 2 \text { at } i \text { th instant, } \\
& \hat{P}_{1_{i, i}}=\text { estimated state error covariance of sensor } 1 \text { at } i \text { th instant, } \\
& \hat{P}_{2_{i, i}}=\text { estimated state error covariance of sensor } 2 \text { at } i \text { th instant. }
\end{aligned}
$$

In this case, the common process noise affecting the target dynamics corresponding to each of the sensors, is assumed to be negligible.

\section{Selection of coordinate system}

Different types of tracking sensors, deployed for target tracking, provide measurement data of the target in different sensor-based reference frames. For example, radar provides measurements in polar coordinate system (range, azimuth and elevation) w.r.t. instrument location. Track data from optical sensors and telemetry antenna image provide angular information (azimuth and elevation) w.r.t. respective sensor locations. However, the vehicle dynamics can be described in different types of coordinate frames (Farina \& Pardini 1980), e.g., (i) polar (range, azimuth and elevation), (ii) rectangular, with $x-y$ plane tangent to the earth surface in radar site and $z$-axis perpendicular to this plane, and (iii) target-oriented Cartesian coordinates, where the $x$-axis is along the direction of azimuth, $y$ axis is along the cross range and $z$ axis is perpendicular to $x-y$ plane. Target tracking in each of the reference frames has its own advantages and disadvantages. Tracking in natural coordinate of measurement is preferred in many applications, as it avoids loss of performance accuracy due to unmodelled errors while transferring data from one reference frame to other. But the main disadvantage of tracking in polar coordinates is incorporation of pseudo-acceleration in all components of motion in Cartesian coordinates, even for constant velocity targets. Tracking filters in target oriented reference frames are decoupled into single-dimension filters, but in this case, the axis system is to be realigned at each scan, when the relative bearing changes. In addition to this, due to the diversified nature of tracking sensors at the Test Range, different tracking algorithms have to be devised for different sensors to track the target in natural coordinate of measurement of the sensors, which results in increased complexity of the filtering and fusion system. From this point of view, tracking in the Cartesian coordinate system has been used at present, which has the advantage of linear and uncoupled dynamic equations. However, in such cases, conversion from polar to rectangular coordinates is necessary for radar measurement, and triangulation of angle-only measurement is necessary for optical sensors and telemetry-antenna-image at every scan.

\section{Noise characterizing methodology adopted at Test Range}

Kalman filtering technique utilizes the noise statistics of the underlying system under consideration (i.e., target dynamics for the present case) and of the measurement process. The optimality of the Kalman filter in minimum mean square error sense depends on the validity of assumption of zero-mean and white Gaussian noise characteristics. The validity of these basic assumptions has been tested by means of exhaustive post-flight data analysis. 
Since the target under consideration for the present case is of non-maneuvering type, the main source of process noise may be attributed to unmodelled dynamics during the sampling interval and wind disturbances. A thorough study showed that selection of sampling interval of $100 \mathrm{~ms}$ and assumption of zero mean white Gaussian process noise meet accuracy requirement of real-time flight safety application. Different techniques available for computation of covariance of process noise and their relative performances have been discussed in literature (Girija \& Raol 2001). These techniques are Heuristic Method, Model Compensation Method, Optimal State Estimate Method by Maybeck, Fuzzy Logic Based Method etc. For practical implementation at Test Range, Heuristic Method has been adopted for computation of process noise variances. It is based on the observation that the Kalman filter performance depends only on the relative magnitude of measurement noise and process noise characteristics and not on their absolute values (Melsa \& Schultz 1967; Girija \& Raol 2001). In this method, process noise covariance is assumed to be dependent on the measurement noise covariance as per the following relationship.

$$
\text { Process noise covariance }=Q=\left(k_{1} \times \sqrt{R} \times e^{-k_{2} \times i \times T}\right)^{2},
$$

where,

$$
\begin{aligned}
R & =\text { Measurement noise variance, } \\
T & =\text { Sampling interval, } \\
i & =1,2, \ldots, N \\
N & =\text { Total number of data points. }
\end{aligned}
$$

However, implementation of this method involves appropriate choice of the proportionality factors $k_{1}$ and $k_{2}$, which is achieved by post-flight data analysis for particular target and sensor type.

The measurement process by the sensors gets corrupted by a number of noise sources, namely geometry of tracking, changes of refractive index of medium of signal propagation, ground multi-path, target orientation, target characteristics, internally generated thermal noise etc. The study of noise characteristics of each of the sensors requires analysis of nature and effect of each of the sources of noise. However, Central Limit Theorem (Papoulis 1991) proves that summed effect of large numbers of independent random variables approximates to Gaussian probability density. For the present application, measurement noise characteristics have been studied by fitting the sensor's track data into ARMA (Auto Regressive Moving Average) model (Papoulis 1991). The residuals are checked for whiteness and the covariance of the residuals is estimated, which is used as the measurement noise covariance. Since the order of the model necessary to fit the data is not known a priori, models with different orders are tried before arriving at the right order based on fit criteria. It has been verified that the noise covariance resulting from the ARMA model conforms to that specified by the designers.

Since measurement data filtering and fusion are carried out in the Cartesian coordinate system, deterministic conversion of noisy polar measurement data for radar and triangulation of noisy angular data from optical sensors and telemetry antenna images are accomplished to compute position in Cartesian frame at every scan. In this case, the measurement variance of the sensors in Cartesian coordinates is computed using the sensor angular accuracy $\left(\rho_{a}\right)$ and estimated range $(\hat{R})$ of the target (since true range data is not available) as given below:

$$
\text { Measurement noise variance in } x, y \text { and } z \text { direction }=\left(\rho_{a} \hat{R}\right)^{2} \mathrm{~m}^{2} \text {. }
$$




\section{Results}

The fusion algorithm has been tested with simulated as well as real post-flight data. Simulation has been carried out with two-dimensional state model of the flight vehicle along $x, y$ and $z$ directions as stated below:

$$
X_{i+1}=\left[\begin{array}{ccc}
F & 0 & 0 \\
0 & F & 0 \\
0 & 0 & F
\end{array}\right] X_{i}+\left[\begin{array}{c}
G \\
G \\
G
\end{array}\right] v_{i},
$$

where

$$
\begin{aligned}
X_{i} & =\text { state vector at } i \text { th instant, } \\
& =\left[\begin{array}{l}
\text { position } \\
\text { velocity }
\end{array}\right] \text { for each of } x, y \text { and } z \text { directions, } \\
F & =\text { state transition matrix for each of } x, y \text { and } z \text { directions, } \\
& =\left[\begin{array}{ll}
1 & T \\
0 & 1
\end{array}\right] \\
T & =\text { sampling interval, } \\
v_{i} & =\text { process noise at } i \text { th instant (zero mean white Gaussian), and } \\
G & =\text { process noise gain matrix for each of } x, y, z \text { directions } \\
& =\left[\begin{array}{c}
T^{2} \\
2 \\
T
\end{array}\right] .
\end{aligned}
$$

Simulation is carried out with two sensors having identical measurement matrices. For simulation with radar measurement data, zero mean white Gaussian noise is injected in range and angle measurement channels of simulated radar data. Subsequently, the noisy measurement data in range, azimuth and elevation is deterministically converted to Cartesian coordinate for filtering and fusing using UD-factor based linear Kalman filter algorithm and state-vector fusion technique. The measurement equation (after deterministic conversion of polar radar measurement data to Cartesian coordinate) is as given below:

$$
Z_{i}^{k}=H X_{i}+\eta_{i}^{k}
$$

where $Z_{i}^{k}$ is the measurement data in Cartesian frame at $i$ th instant from $k$ th sensor (after conversion from polar data), $\eta_{i}^{k}$ is noise affecting the measurement from $k$ th sensor at $i$ th instant. The initial position and velocity of target are $200 \mathrm{~m}, 10 \mathrm{~m} / \mathrm{s}$ in $x$-direction, $-100 \mathrm{~m},-10 \mathrm{~m} / \mathrm{s}$ in $y$-direction and $100 \mathrm{~m}, 10 \mathrm{~m} / \mathrm{s}$ in $z$-direction respectively. The sampling time chosen is $0 \cdot 1 \mathrm{~s}$. Total 100 simulations are carried out for each of the following cases and performance of the fusion algorithm is evaluated in terms of squared position error.

Case I: Simulation is carried out by varying standard deviation of process noise from $0.3 \mathrm{~m} / \mathrm{s}^{2}$ to $10 \mathrm{~m} / \mathrm{s}^{2}$. The standard deviation of noise in range and angle measurement are $10 \mathrm{~m}, 5$ arc min for sensor 1 and $20 \mathrm{~m}, 5$ arc min for sensor 2 respectively. The results of simulation are shown in figure 5a. It is evident from the result that, although the squared position error increases as a function of process noise, the fused result is always in between the sensors data. 

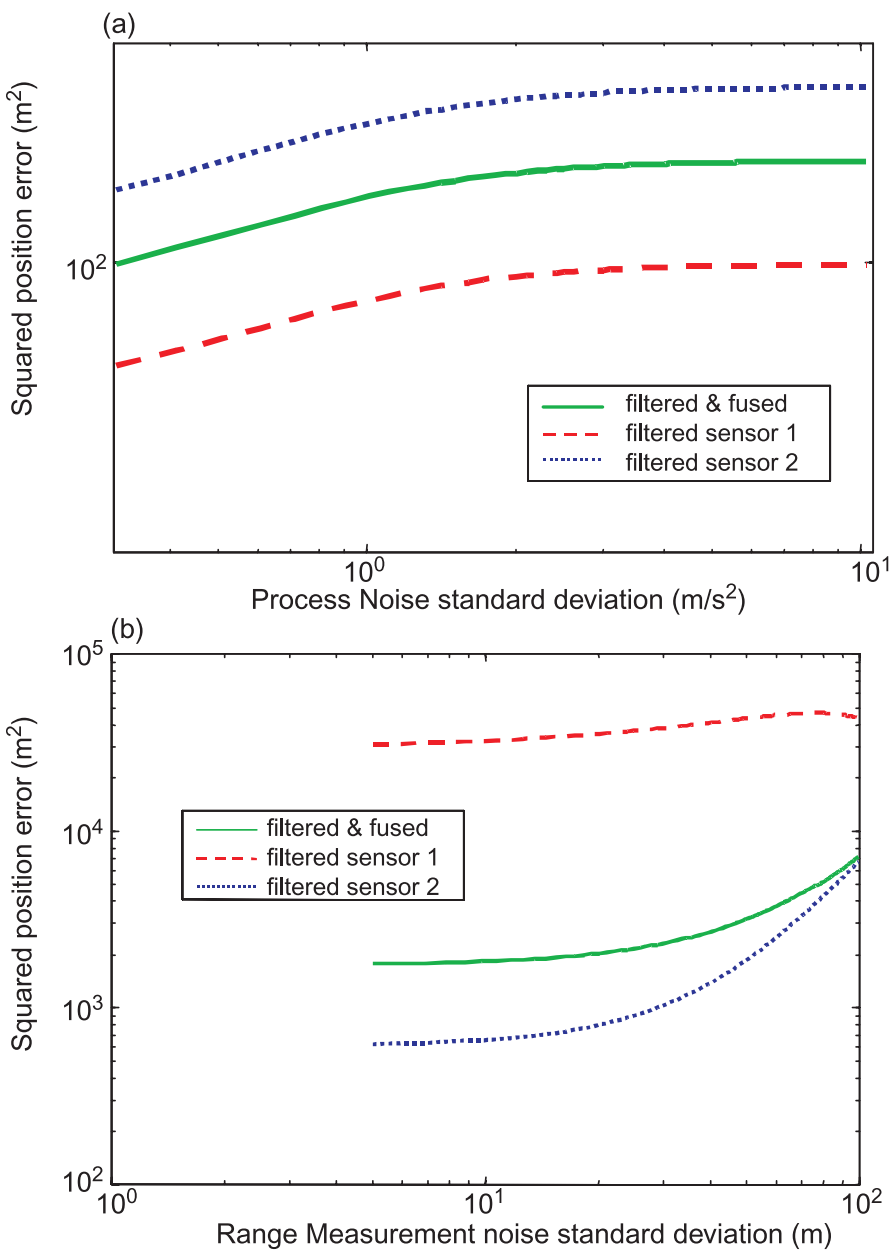

(c)

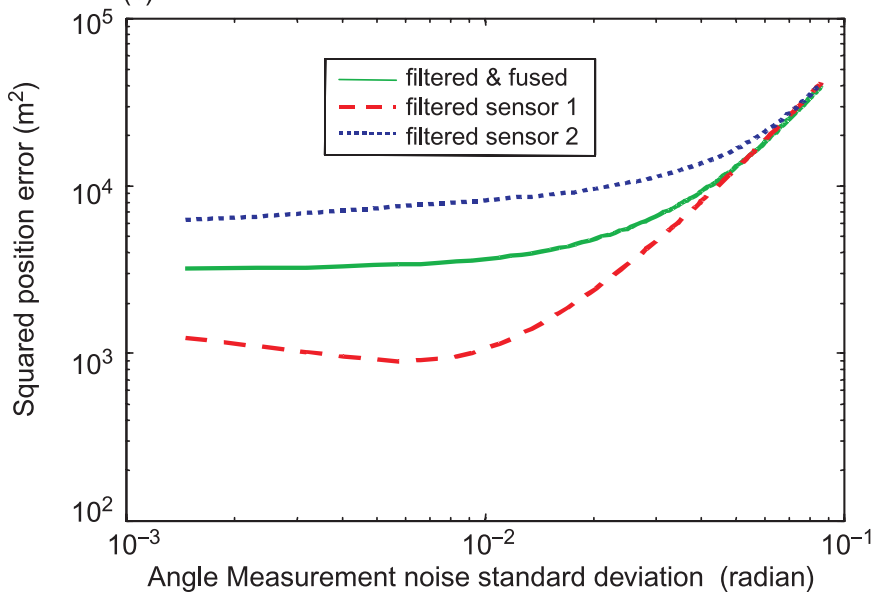

Figure 5. Result with simulated data. 
Case II: In this case, process noise standard deviation is $0.3 \mathrm{~m} / \mathrm{s}^{2}$, angle measurement standard deviation for sensor 1 is 5 arc deg and for sensor 2 is 5 arc min. The simulation is run by varying standard deviation of error in range measurement from $5 \mathrm{~m}$ to $100 \mathrm{~m}$. Figure $5 \mathrm{~b}$ shows the result of simulation.

Case III: Figure 5c brings out the result of simulation by varying standard deviation of angle measurement noise from 5 arc minute to 5 arc degree. In this case, the process noise standard deviation is $0.3 \mathrm{~m} /{ }^{2}$, range measurement error standard deviation for sensor 1 is $50 \mathrm{~m}$ and for sensor 2 is $100 \mathrm{~m}$.

Figures $5 \mathrm{~b}$ and $\mathrm{c}$ show that as measurement error is increased (in range and angle) the squared position error increases, but the fused data are always in between the results of the two sensors.

The result of fusion using real sensor (two radars of similar type) data of a helicopter sortie is shown in figure 6 in terms of fused as well as filtered sensor position data and error of fused
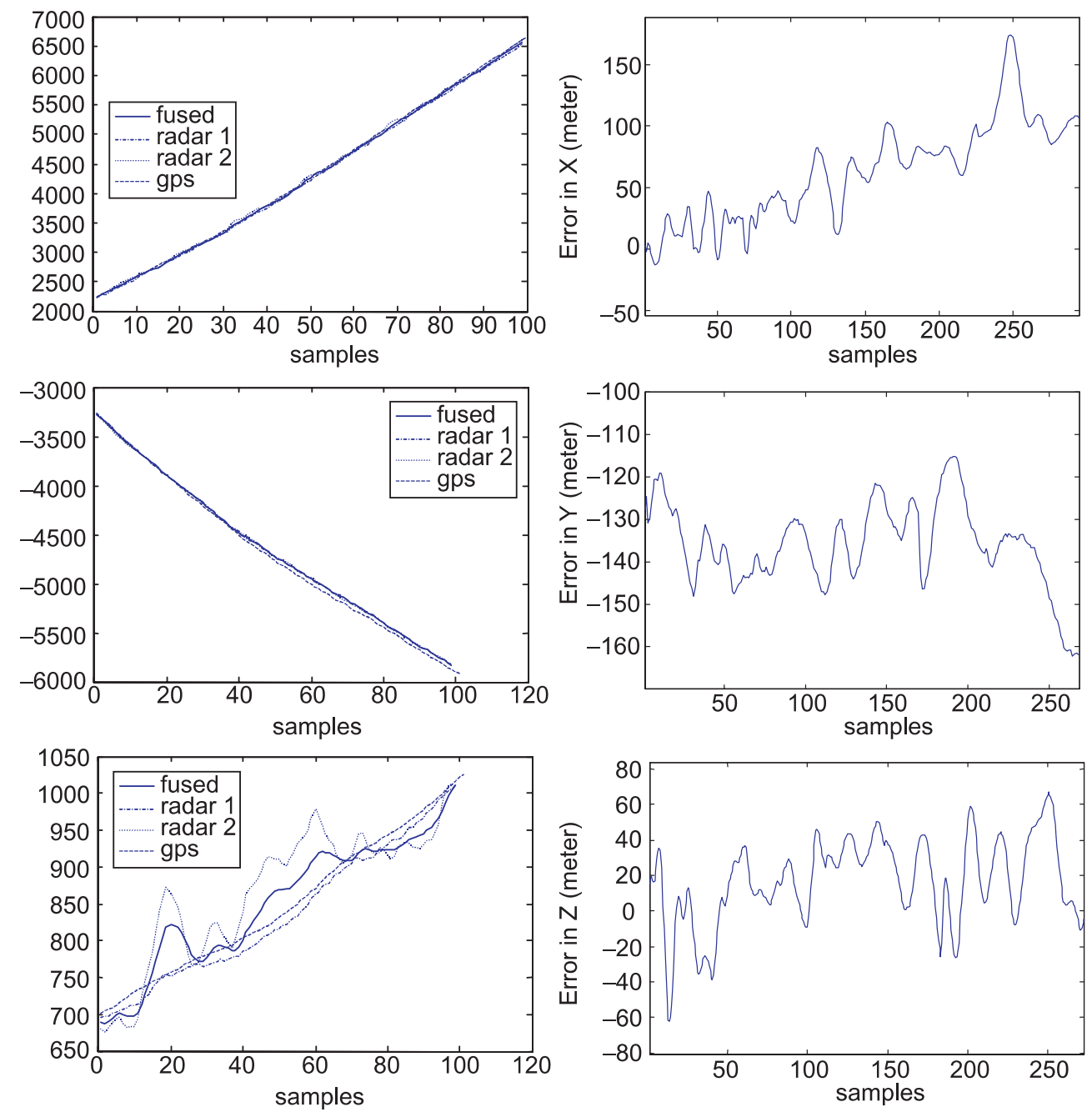

Figure 6. Result with real data. 
position w.r.t. a reference sensor data. GPS (Global Positioning System), being a consistent, reliable and all-weather navigation system, has been considered the reference for computing error of the fused data. The error of the fused data of two radars w.r.t. GPS data shows that the error is within the acceptable limit for real-time flight safety monitoring and decision making purpose.

\section{Conclusion}

Performance of the state-vector fusion technique adopted at the Integrated Test Range, Chandipur for real-time application has been discussed in the present paper. Results with simulated as well as real data show that the fusion algorithm efficiently uses two sensor data sets to provide estimation of target dynamics, which is statistically better (in terms of estimation error) than that of the individual sensors. In state-vector fusion technique, although a separate Kalman filter is required for each of the sensors, it is ascertained from simulation and practical experience that the algorithm can generate fused state estimate with data update rate of $100 \mathrm{~ms}$, after processing twelve sensors data at every time instant. The algorithm is presently being used for real-time mission mode and performs satisfactorily.

The authors express their heartfelt gratitude to Mr S C Narang for his encouragement and advice during the course of the work. The authors are also grateful to Dr J R Raol and Dr G Girija, National Aerospace Laboratories (NAL), Bangalore for their active participation and effective interaction with ITR in connection with the work. Special thanks are extended to the members of the Technical Expert Committee and Human Resource Development Group of ITR for their kind cooperation during processing the Paper. Last, but not the least, the direct and indirect contributions of all colleagues of Flight Safety Group of ITR are acknowledged with appreciation.

\section{List of symbols}

$$
\begin{aligned}
& F \quad(n \times n) \text { state transition matrix for } x, y, z \text { direction } \\
& {\left[\begin{array}{ll}
1 & T \\
0 & 1
\end{array}\right] \text { for second order model; }} \\
& G \quad \text { process noise gain matrix for each of } x, y \text { and } z \text { directions } \\
& =\left[\begin{array}{c}
T^{2} / 2 \\
T
\end{array}\right] \text { for two-dimensional constant velocity model; } \\
& H \quad(m \times n) \text { measurement matrix } \\
& =\left[\begin{array}{llllll}
1 & 0 & 0 & 0 & 0 & 0 \\
0 & 0 & 1 & 0 & 0 & 0 \\
0 & 0 & 0 & 0 & 1 & 0
\end{array}\right] \text { for position measurement in } x, y \text {, and } z \text { directions; } \\
& \hat{P}_{1_{k, k}} \quad \text { estimated state error covariance marix at } k \text { th instant based on sensor-1 } \\
& \text { measurement; } \\
& \hat{P}_{2_{k, k}} \quad \text { estimated state error covariance marix at kth instant based on sensor-2 } \\
& \text { measurement; }
\end{aligned}
$$




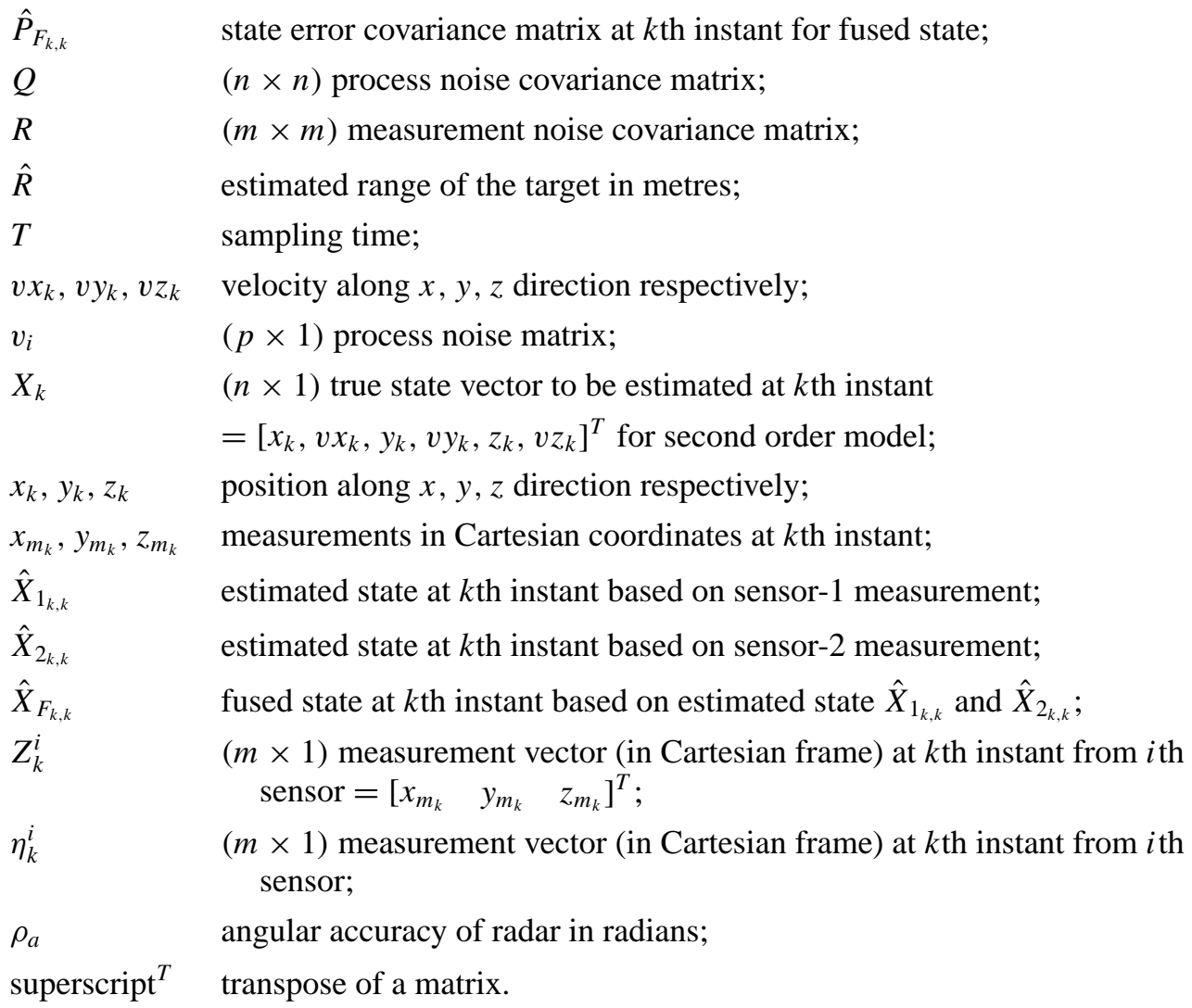

\section{References}

Banerjee P, Raj R A 2000 Multi-sensor data fusion strategies for real time application in test and evaluation of rockets/missiles system. IEEE Proc. : 723-728

Farina A, Pardini S 1980 Survey of radar data-processing techniques in air-traffic control and surveillance systems. Inst. Elect. Electron. Proc. 127: 190-204

Gan Q, Harris C J 2001 Comparison of two measurement fusion methods for Kalman-filter-based multi-sensor data fusion. IEEE Trans. Aerosp. Electron. Syst. 37: 273-280

Girija G, Raol J R 2001 Evaluation of adaptive Kalman filtering methods for target tracking applications. AIAA 2001-4106, pp 1-11

Girija G, Raj R A, Raol J R, Kashyap S 2000 Tracking filter and multi-sensor data fusion. Sādhanā 25: $159-167$

Hall D L 1992 Mathematical techniques in multi-sensor data fusion (Boston, MA: Artech House)

Melsa J L, Schultz D G 1967 State functions and linear control systems (New York: McGraw Hill)

Papoulis A 1991 Probability, random variables, and stochastic processes (New York: McGraw-Hill)

Saha R K 1996 Effect of common process noise on two-track fusion. J. Guidance, Control \& Dynamics 19: 829-835 